DOI 10.17816/transsyst20184262-72

\author{
(C) F. Qin, Y. Lin, D. Lu
}

National Maglev Transportation Engineering R\&D Center, Tongji University

(Shanghai, China)

\title{
HARDWARE-IN-THE-LOOP SIMULATION OF HIGH-SPEED MAGLEV TRANSPORTATION FIVE-SEGMENT PROPULSION SYSTEM BASED ON DSPACE
}

\begin{abstract}
Aim: For exploring and testing the key technology of high-speed maglev transportation propulsion control system, this paper designs and establishes a hardware-inthe-loop (HIL) real-time simulation system of the high-speed maglev transportation fivesegment propulsion system.

Materials and methods of the studies: According to the route conditions and propulsion segment division of Shanghai maglev demonstration and operation line, the realtime simulation platform based on dSPACE multiprocessor systems is implemented. The simulation system can achieve the functional simulation of all the high-power related equipment in the 5-segment area, including 8 sets of high-power converter units, 2 sets of medium-power converter units, 2 sets of low-power converter units, five-segment trackside switch stations and long-stator linear synchronous motors. The mathematical models of linear motors and converters are built in MATLAB/Simulink and System Generator, after compiling, they can be downloaded and executed in Field Programmable Logic Array (FPGA). All the interfaces connecting the simulation system to the propulsion control system physical equipment use real physical components as in the field, such as analog I/O, digital I/O, optical signals and Profibus.

Results: By using CPU+FPGA hardware configuration, the simulation steps are greatly shortened and the response speed and accuracy of real-time simulation system are improved. The simulation system can simulate multiple operating modes such as multisegment, multi-vehicle, double-track, double-feeding, step-by-step stator section changeover, and so on. The simulation results show that the maximum speed of the simulation system can reach $500 \mathrm{~km} / \mathrm{h}$.
\end{abstract}

Conclusion: This HIL system can provide detailed real-time on-line test and verification of high speed maglev propulsion control system.

Keywords: Hardware-in-the-loop, real-time simulation, high-speed Maglev transportation, five-segment, propulsion system, dSPACE, FPGA.

\section{INTRODUCTION}

High-speed maglev transportation has the advantages of high speed, low energy consumption, low noise, no pollution and the strong ability to run over steep slopes and sharp curve and so on. So it is a suitable candidate for nextgeneration transport systems. Shanghai maglev demonstration and operation line is the first commercial operation line in the word, which utilizes long stator linear synchronous motor (LSLSM) propulsion and electromagnetic suspension [1]. 
The propulsion system is one of the core technology of high-speed maglev transportation system, which mainly includes propulsion control system, long stator linear synchronous motors, converters and trackside equipment. However, the development and testing of the propulsion control system face many difficulties. First of all, there is currently no long enough line for the propulsion control system to complete the high-speed test; secondly, the controller at the early stage of development inevitably suffers from various deficiencies, in which case high-speed test will bring a lot of danger and the cost will be relatively high [2]. Therefore, hardware-in-the-loop (HIL) real-time simulation is essential.

The purpose of HIL simulation is to simplify the difficulty of system development and accelerate the development and test of the system. The basic idea is to connect the real controller with the rest of the mathematical model in the system to form a semi-physical simulation system. Through the specially designed hardware interface circuit, the mathematical model running in the realtime simulation system receives the control instructions of the real controller and feeds back the operation results to the controller. Therefore, HIL simulation can complete software and hardware testing on the real controller, while reducing experimental costs [3].

In this paper, dSPACE is used to establish the HIL real-time simulation platform of the high-speed maglev transportation propulsion system. Taking Shanghai maglev train demonstration and operation line as a case of application, the simulation system enables the simulation of all converters, linear motors and trackside equipment in the 5-segment area. The simulation results show that the maximum speed of the simulation system can reach $500 \mathrm{~km} / \mathrm{h}$.

\section{SYSTEM OVERALL ARCHITECTURE}

The propulsion HIL real-time simulation system includes the actual control system devices and the power simulation system devices. The configuration principles of the actual devices and the simulation devices are as follows:

$\checkmark$ Power-related parts are implemented by simulation, such as converter units, linear motors, and trackside feed cables, etc.;

$\checkmark$ Weak electricity and control related parts adopt actual devices, such as motor control units and converter control units inside the propulsion control system;

$\checkmark$ The same devices are implemented with 1 to 2 sets of actual devices, and the rest is realized by simulation, such as trackside switch station control units;

$\checkmark$ The interfaces between the simulation devices and the actual devices are configured as much as possible based on the actual interfaces (such as analog signal interfaces, digital signal interfaces, optical fiber 
signal interfaces, Profibus interfaces, etc.), but for the convenience of debugging, some debugging interfaces (such as position signal interface) can be simplified and added.

The high-speed maglev propulsion HIL system uses Shanghai maglev demonstration and operation line as its application background. The total length of Shanghai line is approximately 30 kilometers, with two tracks A (downward) and B (upward). There are two propulsion substations on the whole line, respectively \#1 propulsion substation and \#2 propulsion substation, as shown in Fig. 1. Among them, \#1 propulsion substation is equipped with two sets of highpower converter blocks, and \#2 propulsion substation is equipped with two sets of high-power converter blocks, one sets of medium-power converter blocks and one sets of low-power converter blocks.

The whole line is divided into five propulsion segments (Fig. 1). Among them, the main line is divided into four propulsion segments: the main track PSE2A (red), PSE2B (blue), Longyang Road Station PSE1 (purple), Pudong Airport Station PSE3 (light green); the entire maintenance area is a propulsion segments PSE90 (rose red). Double-feeding is carried out in PSE2A and PSE2B propulsion segments. In the propulsion segments of PSE1, PSE3, and PSE90 operation takes place using single-feeding. The step-by-step method is adopted for the whole line, and each set of converter block is equipped with two sets of converter units, two sets of feeding cables and corresponding switching devices.

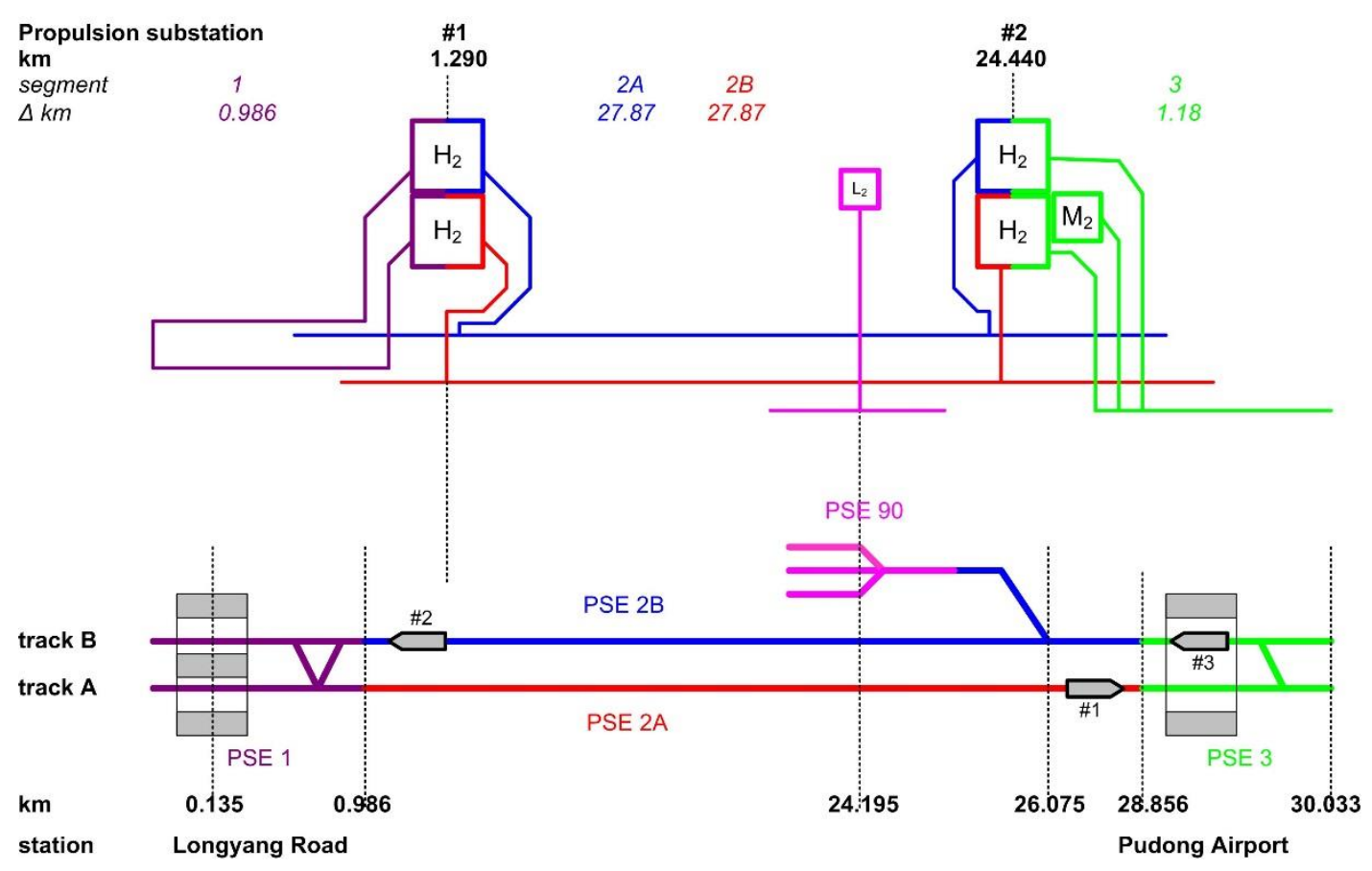

Fig. 1. Overall configuration of the propulsion system

According to the above requirements, the specific configuration of the propulsion HIL simulation system is shown in Fig. 2. 


\section{Propulsion control system}

(Motor control units、Converter control

units、Converter peripheral control units、

Trackside switch station control units)

\section{Position}
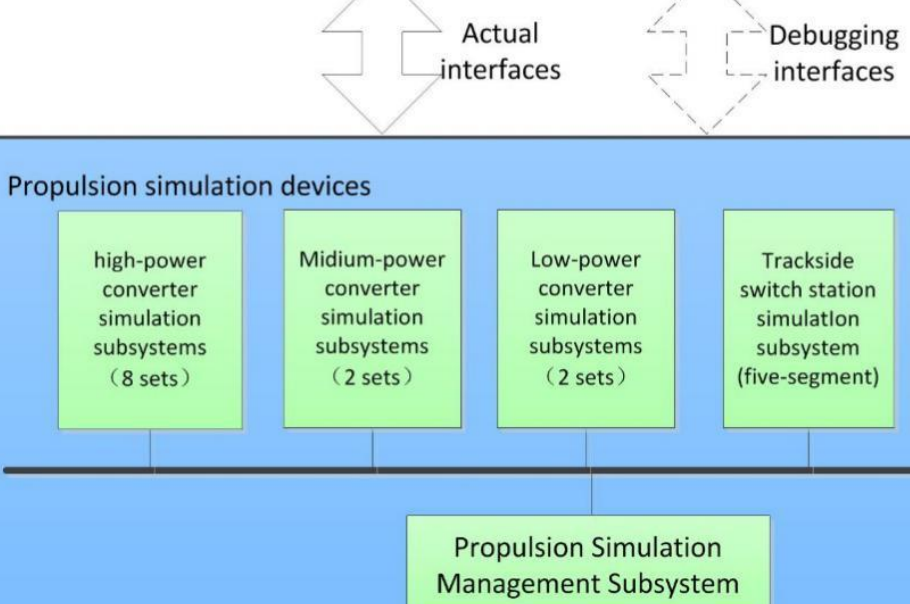

Propulsion simulation devices

converter

simulation

subsystems

(8 sets)
Midium-power
converter
simulation
subsystems
( 2 sets)

(1)

(2 sets)

Propulsion Simulation

Management Subsystem

interfaces

interfaces

Fig. 2. The specific configuration of the propulsion HIL simulation system

\section{Propulsion control system}

In addition to trackside switch station control units, the rest of the propulsion control system uses physical devices, including motor control units (MCU), converter control units (CCU), converter peripheral control units, and the monitoring and diagnostic system. Since there are many trackside switch stations along the line (the average interval is 600 meters) and all trackside switch stations have the same working principle, only two sets of trackside switch station control units use actual devices, and the rest are implemented using simulation systems.

2. Propulsion simulation system

According to the function division, the propulsion simulation system mainly consists of 12 sets of converter simulation subsystems, trackside switch station simulation subsystem and 3 sets of linear motor simulation subsystems.

1) 12 sets of converter simulation subsystems include 8 sets of 15 MVA high-power converter simulation subsystems, 2 sets of 7.5 MVA medium-power converter simulation subsystems and 2 sets of $400 \mathrm{kVA}$ low-power converter simulation subsystems. Each set of converter simulation subsystem can achieve the functional simulation and interface simulation of a neutral point clamp (NPC) three-level backto-back AC-DC-AC converter, input switchgears, input transform, output transform and output switchgears;

2) The trackside switch station simulation subsystem can implement functional simulation and interface simulation of all trackside switch stations and their control units within 5 propulsion segments. As the 
trackside switch stations in each segment exchange information with the motor control units through redundant Profibus networks, so the trackside switch station simulation subsystem of five-segments needs to configure 10 Profibus communication interfaces;

3) The linear motor simulation subsystem is used to simulate the electromagnetic characteristics of the two long stator synchronous linear motors on the left and right sides of each column of the maglev vehicle and the vehicle's motion characteristics. 3 sets of linear motor simulation systems correspond to 3 maglev vehicles.

According to the requirements of the operation mode of the high-speed maglev propulsion system, the propulsion simulation system can realize the simulation of various working modes, such as single-feeding, double-feeding, and step-by-step stator section changeover mode, etc. For this reason, the converter simulation subsystems of different numbers and power levels can be co-simulated with different linear motor simulation subsystems and trackside switch station simulation subsystem. Therefore, the propulsion simulation system can implement different operating modes according to the operating mode of the high-speed maglev system.

In addition, the propulsion simulation system needs to configure the propulsion simulation management subsystem to realize the development and download of the simulation system models, online real-time monitoring and parameter modification. The propulsion simulation management subsystem can also inject faults and detect fault conditions in real-time simulation systems, set up various test cases, and complete the comprehensive testing and verification of the propulsion control systems. The propulsion simulation management subsystem can be made up of one or several host computers.

\section{PROPULSION SIMULATION SYSTEM DESIGN}

In the hardware configuration of the propulsion simulation system, five dSPACE real-time simulators are used to form a multi-processor system to implement the functional simulation and hardware interface simulation of the 5 -segment propulsion power devices. The corresponding relationship between the subsystems of the 5-segment propulsion simulation subsystems and the dSPACE real-time simulators is shown in Fig. 3. 


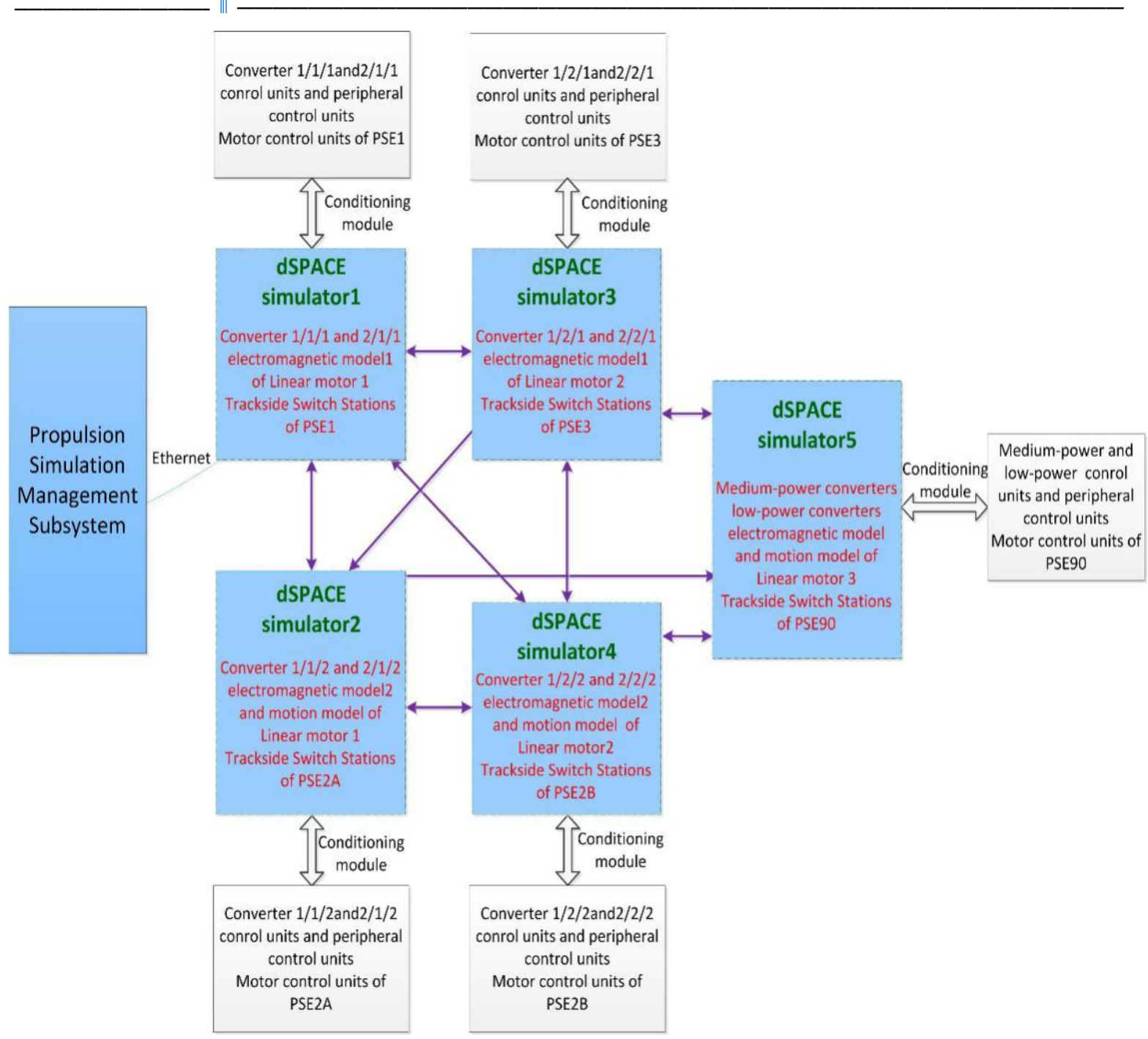

Fig. 3. Hardware configuration of 5-segment propulsion simulation system

Five dSPACE simulators communicate with each other through fiber optics to form a multi-processor system that enables real-time information exchange. The propulsion simulation management subsystem can be connected to any dSPACE simulator through an Ethernet network cable, enabling information exchange with 5 dSPACE simulators. The five dSPACE simulators receive instructions from the converter control units and converter peripheral control units through conditioning modules, and feedback voltage and current information. The conditioning modules are used to transform the actual interfaces of the propulsion control system into the signals that the dSPACE simulator can receive. The five dSPACE simulators receive the commands of trackside switch stations from the motor control units via the Profibus network and feed back the status information. The dSPACE simulator $2 \backslash 4 \backslash 5$ feeds back the position information of the maglev vehicle to the corresponding motor control units through UDP interface respectively, in order to realize the position closed-loop control. 
dSPACE real-time simulators use CPU + FPGA architecture, configured hardware boards includes DS1007 processor board, FPGA board and its piggyback module, Profibus board and D/A board. The DS1007 is connected to other boards via PHS bus and to the propulsion simulation management subsystem via Ethernet interface.

The processor board adopts a dual-core CPU. One of the CPUs is used to implement real-time simulation models with low real-time requirements and external interface models, such as trackside switch station simulation subsystems, trackside Profibus communication, UDP communication, etc. The other CPU mainly undertakes data exchange with the FPGA board, including parameter setting, on-line data observation, and interaction of simulation data, etc.

The FPGA board has $100 \mathrm{MHz}$ clock period. It can be configured with 32 digital input/output channels and 12 analog input/output channels. The use of FPGA's high-speed computing capability can achieve the computational tasks of mathematical models with high real-time requirements, such as converters and long stator linear motor mathematical models. Because each FPGA board has a limited number of digital I/O channels and analog I/O channels, each dSPACE simulator requires the configuration of four FPGA boards to implement the mathematical models of two sets of converters and a set of long stator linear motor.

In view of the large number, large-scale and complex features of the propulsion system equipment, a decoupled modeling algorithm was used [4-5]. Firstly the independent model of each simulation subsystem was established using MATLAB/SIMULINK and System Generator software, such as the converter rectifier side mathematical model and inverter side mathematical model, single-feeding long stator synchronous linear motor mathematical model, double-feeding long stator synchronous linear motor mathematical model, etc. Secondly the energy storage element connected with the converter switching element is equivalent to a voltage source or a current source, then an equivalent decoupling circuit between subsystems is obtained. The independent equivalent circuits of all subsystems are cascaded into the equivalent circuit of the propulsion simulation system as a whole, which is also the overall mathematical model of the propulsion simulation system.

\section{SIMULATION RESULTS}

The propulsion simulation system and the propulsion control system make up the hardware-in-the-loop real time simulation system. The photos are shown in the Fig. 4, 5. 


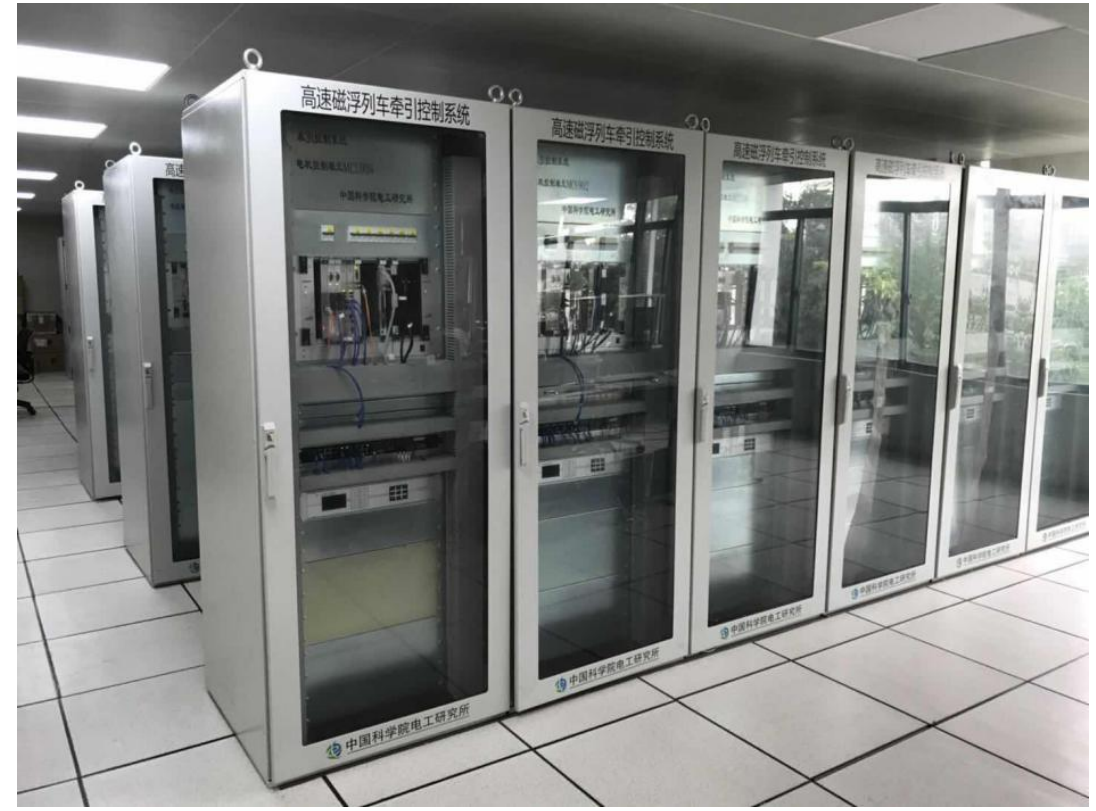

Fig. 4. Propusion control system

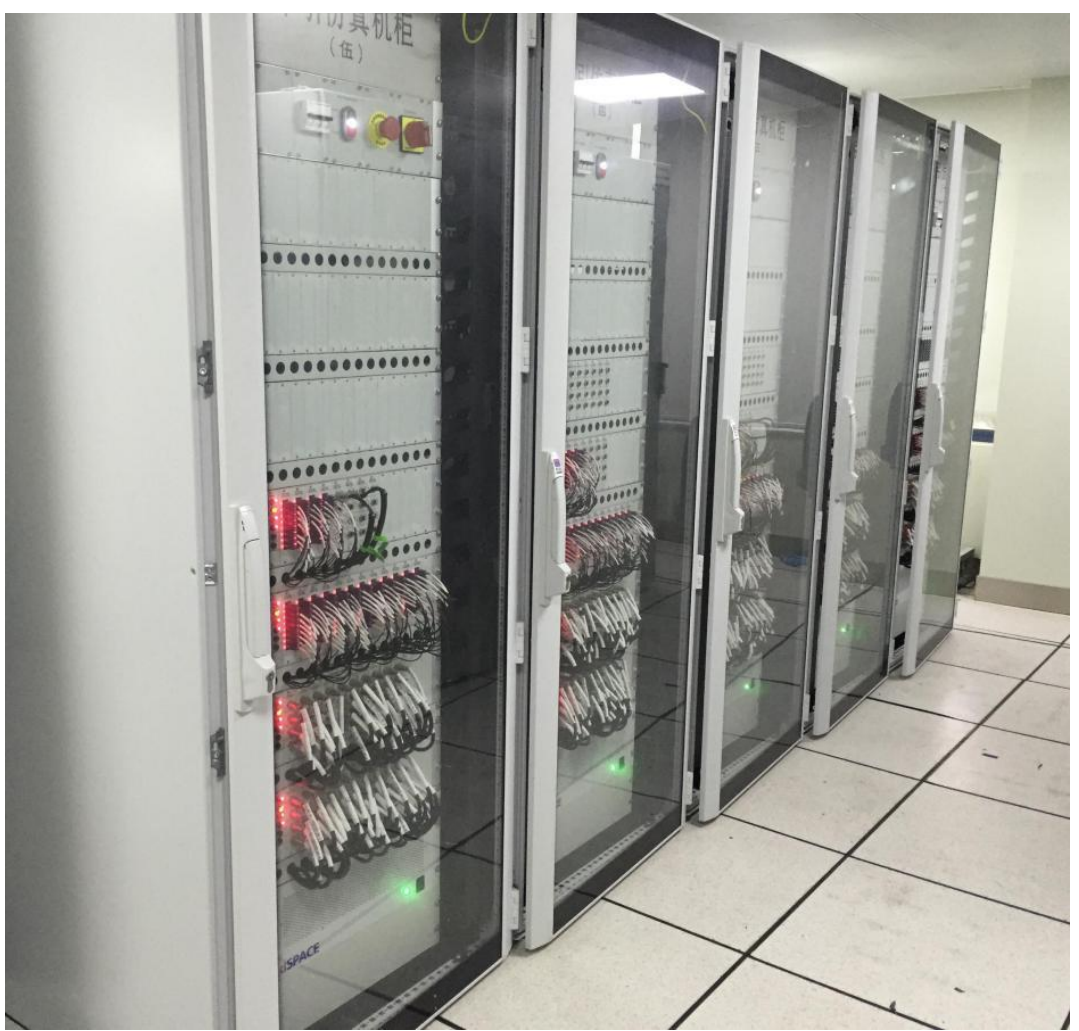

Fig. 5. Propusion simulation system

The HIL system can complete the simulation test of various operation modes, including multi-segment, multi-vehicle, double-track, single-feeding and double-feeding method. The propulsion control system uses rotor field oriented 
control with constant excitation and stator current $\mathrm{d}$ axis component constant to zero, and realizes position, speed, and current three closed-loop control. The simulation step of the whole propulsion simulation system is 100 microseconds, but the operation time of the mathematical model of the converter and the linear motor in FPGA is only 400 ns. Thus, it can be seen that the hardware architecture of CPU+FPGA can guarantee the real time of the model operation to the maximum degree, so as to meet the real time requirement of the whole HIL system.

In the case of four sets of high power converters with double-feeding, the maximum operating speed of an unloaded maglev vehicle can reach $500 \mathrm{~km} / \mathrm{h}$. The Fig. 6 and Fig. 7 show the speed and acceleration curves.

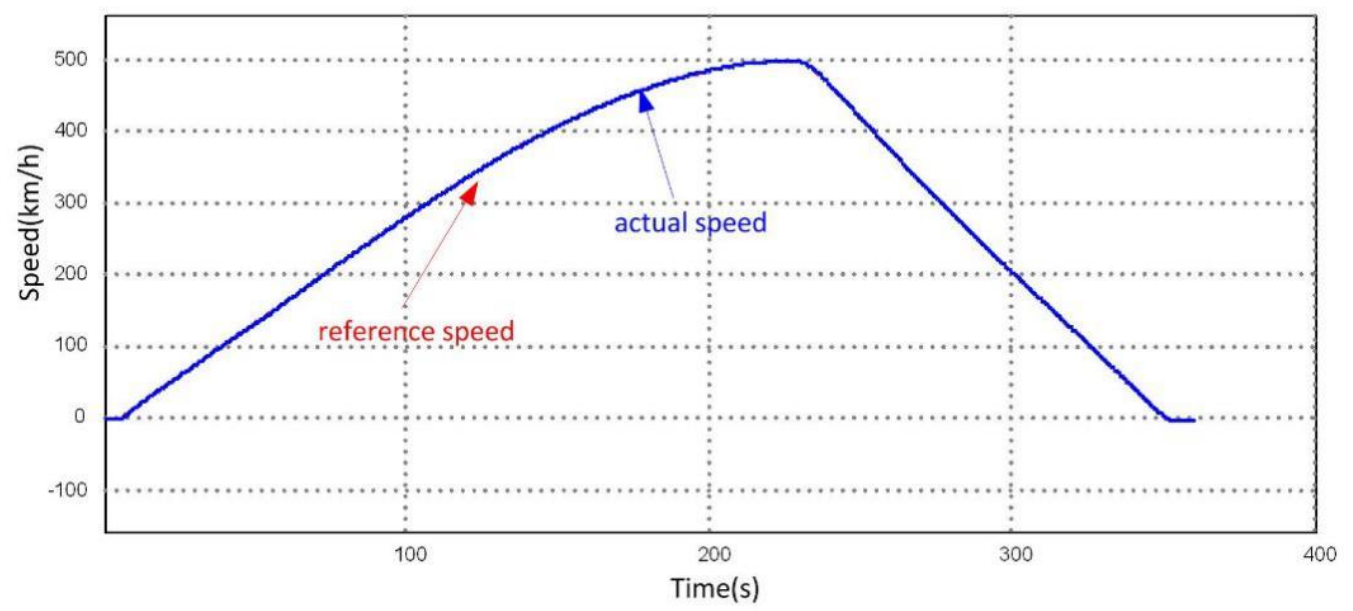

Fig. 6. Speed curve with a maximum velocity of $500 \mathrm{~km} / \mathrm{h}$

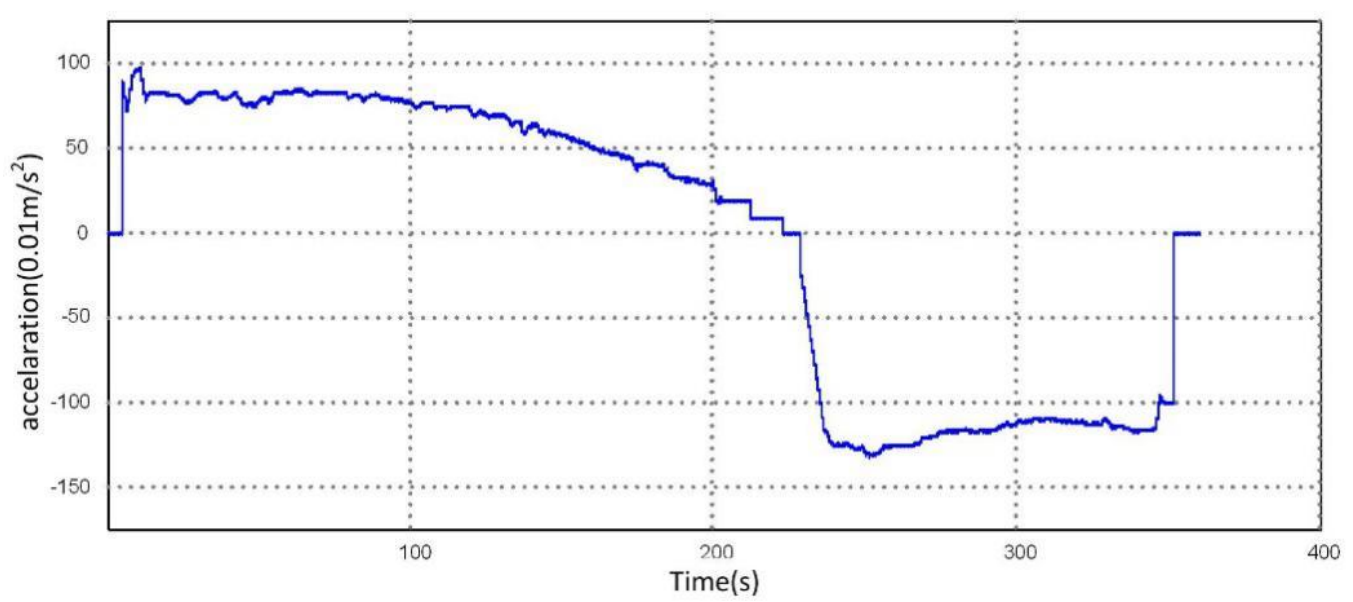

Fig. 7. Acceleration curve with a maximum velocity of $500 \mathrm{~km} / \mathrm{h}$

The velocity of the vehicle is accelerated from 0 to $500 \mathrm{~km} / \mathrm{h}$ by maximum acceleration speed $1 \mathrm{~m} / \mathrm{s}^{2}$ and decelerated by maximum acceleration $1.2 \mathrm{~m} / \mathrm{s}^{2}$. The total mileage is $27.5 \mathrm{~km}$. As can be seen from the speed curve, the vehicle actual speed follows the given speed curve. 
In the case of double-feeding, the speed and acceleration curves of the maglev vehicle with full load and a maximum operating speed of $430 \mathrm{~km} / \mathrm{h}$ are shown in the figures below (Fig. 8, 9).

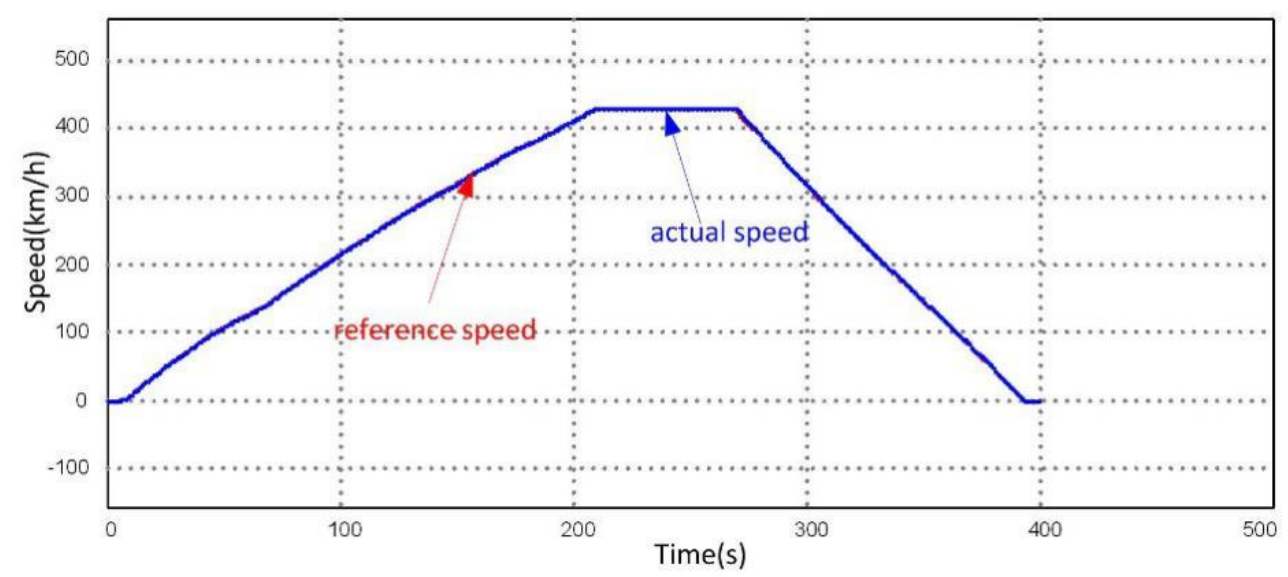

Fig. 8. Speed curve with a maximum velocity of $430 \mathrm{~km} / \mathrm{h}$

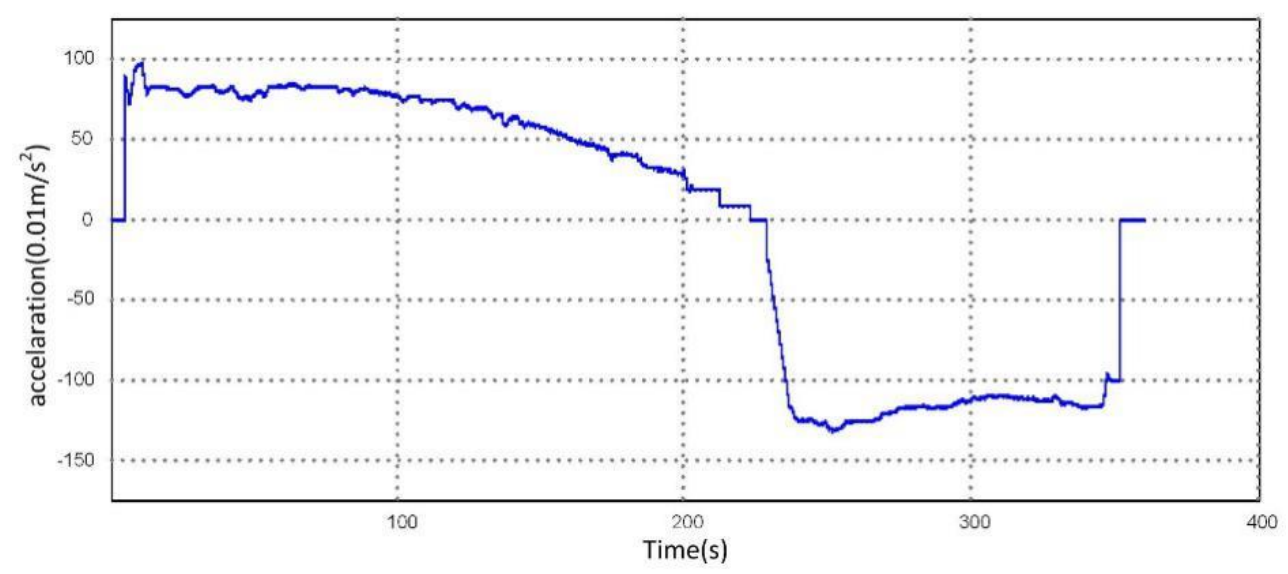

Fig. 9. Acceleration curve with a maximum velocity of $430 \mathrm{~km} / \mathrm{h}$

The vehicle runs at a constant maximum speed for 58 seconds. The total mileage is $27.496 \mathrm{~km}$. Maximum acceleration at deceleration is $-1.3 \mathrm{~m} / \mathrm{s}^{2}$. The actual speed is basically consistent with the given speed curve.

\section{CONCLUSION}

This paper has established a HIL simulation system for high-speed maglev transportation propulsion system based on Shanghai maglev demonstration and operation line. dSPACE multiprocessor system is used to implement the functional simulation and hardware interface simulation of the 5 -segment propulsion power-related devices. The simulation results verified that the real-time simulation is successful and the function of the propulsion control system has been verified. This system can provide detailed test and verification for the propulsion control system and can also be used for the planning and 
design of high-speed maglev transportation, the hardware and software development in the propulsion control system, and system integration.

\section{ACKNOWLEDGEMENT}

This paper is supported by the National Key R\&D Program of China, Research on Simulation Verification and Design Optimization of Key Technologies for High Speed Maglev Transportation System (No. 2016YFB1200602-02).

\section{References}

1. Wu X. Maglev train. 1st ed. Shanghai: Shanghai Sciences and Technology Press; 2003.

2. Terwiesch P, Keller T, Scheiben E. Rail vehicle control system integration testing using digital hardware-in-the-loop simulation. IEEE Transactions on Control Systems Technology, 1999;7(3):352-362. doi: 10.1109/87.761055

3. Li Y, Li Ming S, Hui Z, Du Y. Real-Time Simulation of Linear Synchronous Motor in Hardware-In-Loop Test System. Proceeding of The 13th International Conference on Electrical Machines and Systems (ICEMS2010); 2010 Oct.10-13; Incheon, South Korea. IEEJ Transactions on Industry Applications. 2010;131(2):1520-1523. doi: 10.1541/ieejias.131.nl2_5

4. Byoung-Kuk L, Ehsami M. A simplified functional simulation model for three-phase voltage-source inverter using switching function concept. IEEE Transactions on Industrial Electronics. 2001;48(4):309-321. doi: 10.1109/41.915410

5. Li Y, Li Ming S, Hui Z. Real-time simulation of linear synchronous motor drives with AC-DC-AC converters. Proceeding of he 6th IEEE Conference on Industrial Electronics and Applications; 2011 June 21-23; Beijing, China. IEEE 2011. p. 680685. doi: 10.1109/iciea.2011.5975672

\section{Information about the authors:}

Feng Qin, PhD, assistant researcher; Caoan Highway 4800, Shanghai, China;

ORCID: 0000-0001-9945-1669;

E-mail: qinfeng@ tongji.edu.cn

Ying Lin, master, assistant researcher;

ORCID: 0000-0002-2270-272X;

E-mail: carfieldlin@tongji.edu.cn

Diqiang $\mathrm{Lu}, \mathrm{PhD}$, assistant researcher;

ORCID: 0000-0003-2688-9023;

E-mail: ludiqiang@tongji.edu.cn

\section{To cite this article:}

Qin F, Lin Y, Lu D. Hardware-in-the-loop Simulation of High-Speed Maglev Transportation Five-Segment Propulsion System Based on DSPACE. Transportation Systems and Technology. 2018;4(2):62-72. doi: 10.17816/transsyst20184262-72 